\title{
A locking-free maximum-entropy meshfree method for the simulation of shear-deformable plates based on a mixed variational formulation
}

\author{
J. S. Hale ${ }^{\text {a }}$ P. M. Baiz Villafranca ${ }^{a}$ \\ ${ }^{a}$ Imperial College, Department of Aeronautics, Roderic Hill Building, South Kensington, London, SW7 \\ $2 A Z$
}

\begin{abstract}
The problem of shear-locking in the thin-plate limit is a well known issue that must be overcome when discretizing the Reissner-Mindlin plate equations. In this paper we present a shear-locking-free method utilising meshfree maximum-entropy basis functions and rotated Raviart-Thomas-Nédélec elements within a mixed variational formulation. The formulation draws upon well known techniques in the finite element literature. Due to the inherent properties of the maximum-entropy basis functions our method allows for the direct imposition of Dirichlet (essential) boundary conditions, in contrast to methods based on moving least squares basis functions. Furthermore, our method requires only first-order consistent basis functions and there is no requirement to calculate second-order derivatives. We present benchmark problems that demonstrate the accuracy and performance of the proposed method.
\end{abstract}

Keywords: meshfree, meshless, maximum entropy principle, shear-deformable plates, shear-locking

\section{Introduction}

A common problem encountered in numerical formulations of the MindlinReissner plate bending equations is the phenomenon of shear-locking. This problem manifests itself as an overly stiff system as the plate thickness $\bar{t} \rightarrow 0$ and can be attributed to the inability of the numerical approximation functions to be able to represent the Kirchoff mode [1].

The shear-locking problem was first studied extensively in the context of the Finite Element Method (FEM). One of the primary motivations for using the Mindlin-Reissner plate theory over the Kirchoff theory from a numerical standpoint is the ease of constructing finite element subspaces of $H^{1}\left(\Omega_{0}\right)$, or

Email addresses: j.hale09@imperial .ac.uk (J. S. Hale), p.m.baiz@imperial .ac.uk (P. M. Baiz Villafranca) 
$\mathrm{C}^{0}$ interelement continuity, as opposed to the requirement of constructing a subspace of $H^{2}\left(\Omega_{0}\right)$, or $C^{1}$ interelement continuity for the Kirchoff theory [1]. The latter requires the use of more exotic finite elements, such as the Argyris triangle [2], as opposed to the familiar and relatively easy to implement loworder Lagrangian elements for the former. However it is well known that using low-order Lagrangian elements for all of the displacement fields will result in shear-locking in the thin plate limit [1].

Various remedies have been introduced in the finite element literature to overcome this problem, including, but not limited to; selective reduced integration methods [3], the Assumed Natural Strain (ANS) or Mixed Interpolation of Tensorial Components (MITC) method eg. [4, 5, 6], the Enhanced Assumed Strain (EAS) method eg. [7, 8], and the Discrete Shear Gap method eg. $[9,10]$. The underlying mathematical reasoning for these methods can be found in analysis via a mixed variational formulation such as the HellingerReissner or Hu-Washizu principle [11] where a combination of stresses, strains and displacements are treated as independent variational quantities, see eg. [8] for mixed treatment of EAS-type methods and [1] for mixed treatment of ANS-type methods.

In the meshfree literature various distinct procedures have been introduced to overcome the shear-locking problem:

Increase consistency of approximation This method can be seen as a form of $p$-refinement. Increasing the consistency of the approximating functions means that the Kirchoff mode can be better represented and thus locking is partially alleviated. However, spurious oscillations can occur in the shear strains and the convergence rate is non-optimal [12]. Furthermore, high-order consistency meshless basis functions are more computationally expensive, firstly due to the larger number of nodes that must be in the nodal support to ensure invertibility of the moment matrix, and secondly because of the increased bandwidth of the assembled stiffness matrix [12]. Works using this approach in the $h p$-cloud context include those by Garcia et. al. [13] for shear-deformable plates and Mendonça et. al. [14] for shear-deformable beams. In the context of the Element-Free Galerkin (EFG) method this method has been used by Choi and Kim [15].

Matching fields This approach was originally introduced by Donning and Liu [16] using cardinal spline approximation and later in the context of the EFG method by Kanok-Nukulchai et. al. [12]. In this approach the Kirchoff mode is matched exactly by approximating the plate rotations using the derivatives of the basis functions used to approximate the transverse displacement. More recently the matching fields approach has been used by Bui et. al. [17]. However, as proven by Tiago and Leitão [18] using either the $m$-consistency condition II of Liu et. al. [19], or the Partition of Unity concept of Babuška and Melenk [20], the global system of equations are singular because of a linear dependency in the basis functions for the rotations. 
Nodal Integration These schemes essentially try to mimic the reduced integration method used in Finite Elements. Beissel and Belytschko [21] showed that meshless reduced integration techniques can suffer from spurious modes, similar to their FE counterparts. Some form of stabilisation is required to neutralise these problems. Wang and Chen [22] introduced Smoothed Conforming Normal Integration (SCNI), a form of curvature smoothing, to alleviate locking.

Change of Variables In the analysis of Timoshenko beams, Cho and Atluri [23] use a change of dependent variables, from transverse displacement and rotation to transverse displacement and shear strain on the plate mid-surface to alleviate shear-locking. This approach has been extended to plates by Tiago and Leitão [18], but requires at least second-order consistent shape functions.

Mixed Formulation In the mixed formulation fields such as stresses, strains and pressures are treated as independent variational quantities in the weak form. In the field of meshless methods this approach has primarily been applied to the problem of locking in nearly incompressible elasticity where Poisson's ratio $v$ approaches $1 / 2$. Vidal et. al. [24] used diffuse derivatives to construct pseudo-divergence-free approximations for the displacement that would satisfy the incompressibility constraint a priori. González et. al. enriched the displacement approximation in a Natural Element Method formulation [25]. The B-bar method from the FE literature [26] was introduced into the EFG method by Recio et. al. [27]. Recently Sukumar et. al. and Ortiz et. al. [28, 29] constructed a method where the pressure variables are eliminated by calculating volume-averaged pressures across domains attached to a node to formulate a generalised displacement method.

In this work an approach based on the mixed formulation of the ReissnerMindlin plate equations is used for the first time within the context of a meshfree method to solve the problem of shear-locking. We use first order consistent maximum-entropy basis functions $[30,31]$ combined with lowestorder rotated Raviart-Thomas-Nédélec [32,33] finite elements to construct a hybrid meshfree/finite element method that is free of shear-locking. Due to the inherent properties of the maximum-entropy basis functions our method possesses a so-called 'weak' Kronecker delta property and positive mass matrix amongst other advantages. We show that the approach alleviates shearlocking for a variety of numerical test problems. Finally we show the convergence and locking-free property of the proposed method numerical experiments for some test problems.

The outline of the paper is as follows; In section 2 we give an introduction to maximum-entropy basis functions. In section 3 we give an overview of the Reissner-Mindlin equations and derive the mixed discrete formulation. In section 4 we present numerical examples for various benchmark problems and show the suppression of shear-locking. Finally, we end with some brief 
remarks in section 5. In appendix Appendix A we discuss the construction of Raviart-Thomas-Nédélec elements of lowest order and in appendix Appendix $\mathrm{B}$ we discuss conforming transforms between reference and global elements.

\section{Maximum-entropy basis functions}

\subsection{Background}

Shannon's [34] principle of Maximum-Entropy was postulated by Jaynes [35] as a measure of uncertainty of a probability distribution. The Shannon entropy $H$ of a discrete probability distribution $p=\left\{p_{1}, p_{2}, \ldots, p_{n}\right\}$ associated with $n$ events $x=\left\{x_{1}, x_{2}, \ldots, x_{n}\right\}$ is defined by:

$$
H(\boldsymbol{p})=E(-\ln \boldsymbol{p})=-\sum_{i=1}^{n} p_{i} \ln p_{i}
$$

where $E$ is the usual expectation function. Jaynes proposed that in the case when insufficient information is available, the least-biased probability distribution $\boldsymbol{p}$ is the one that maximises Shannon's measure of entropy $H(\boldsymbol{p})$ subject to the known prior information about the distribution [35].

Shannon originally introduced his measure of uncertainty in the context of communication theory [34], and it has been widely applied in fields as diverse as biology [36] and machine learning [37]. In the context of constructing a meshfree approximation scheme, Sukumar [38] established that the basis functions $\phi_{i}$ could be identified as discrete probabilities $p_{i}$ associated with the nodes located at $x_{i}$. In the words of Sukumar et. al. [39]: "the basis function value $\phi_{i}$ is viewed as the probability of influence of a node $i$ located at a point $x_{i} . "$

In the first paper by Sukumar [38] the principle of maximum entropy was used to generate interpolants on polygonal convex domains.

Arroyo and Ortiz [30] introduced a modified entropy functional of the form:

$$
H(\boldsymbol{\phi}, \boldsymbol{x})=-\sum_{i=1}^{n} \phi_{i}(\boldsymbol{x}) \ln \phi_{i}(\boldsymbol{x})-\beta(\boldsymbol{x}) \sum_{i=1}^{n} \phi_{i}(\boldsymbol{x})\left\|x_{i}-\boldsymbol{x}\right\|^{2}
$$

where $\beta(x) \in \mathbb{R}$ is a parameter that can be varied to adjust the support width of the meshless basis functions. When $\beta \rightarrow \infty$ the Delaunay (linear finite element) interpolant is obtained, and for $\beta \rightarrow 0$ Shannon's standard measure of entropy is recovered. Adjustment of the parameter $\beta(x)$ across the domain can allow for seamless transition between regions discretised using finite elements and regions discretised with meshfree basis functions.

Later, in the most general approach, Sukumar and Wright [31] proposed the use of a relative entropy functional which allows the choice of any sufficiently smooth prior weight function $w_{i}$ associated with each node $i$ :

$$
H(\boldsymbol{\phi}, \boldsymbol{w})=-\sum_{i=1}^{n} \phi_{i}(\boldsymbol{x}) \ln \left(\frac{\phi_{i}(\boldsymbol{x})}{w_{i}(\boldsymbol{x})}\right)
$$


It can easily be shown that for the choice of a Gaussian Radial Basis Function (RBF) for the prior weight function $w_{i}(\boldsymbol{x})=\exp \left(-\beta(\boldsymbol{x})\left\|\boldsymbol{x}_{i}-\boldsymbol{x}\right\|^{2}\right)$ the modified entropy functional eq. (2) of Arroyo and Ortiz may be recovered [31]. In this paper we use the $C^{2}$ quartic spline as our prior weight function:

$$
w_{i}(\bar{r})= \begin{cases}1-6 \bar{r}^{2}+8 \bar{r}^{3}-3 \bar{r}^{4}, & 0 \leq \bar{r} \leq 1 \\ 0, & \bar{r}>1\end{cases}
$$

where $\bar{r}=\left\|x_{i}-x\right\| / \rho_{i}$ and $\rho_{i}$ is the support radius of node $i$.

\subsection{Properties}

Maximum Entropy basis functions have various advantageous properties over the more commonly used MLS type basis functions which have seen wide application in the Element-Free Galerkin (EFG) method [40]. These properties include [30]; variation diminishing property (roughly speaking, the approximation is not more oscillatory than the data that it approximates), $C^{\infty}$ continuity [41], positivity $\phi_{i} \geq 0 \forall i$ which leads to a positive mass matrix, and a 'weak' Kronecker delta property.

We will expand on the weak Kronecker delta property here. It is wellknown that the MLS shape functions do not satisfy the Kronecker delta property and therefore the trial and test function spaces cannot be built to satisfy the Dirichlet (essential) boundary conditions a priori [42]. Two solutions present themselves using MLS type approximation schemes, an excellent overview is given by Huerta et. al. [42]; the first is to use a modified weak form, such at the method of Lagrange multipliers, penalty method or Nitsche's method [43], which enforces the essential boundary conditions as part of the weak form, or alternatively blend meshfree approximations with Lagrangian finite elements near the boundary [44, 42].

For maximum entropy basis functions there is no need to resort to any special methods to enforce Dirichlet boundary conditions. Arroyo and Ortiz [30] prove that for a node set $X$ with convex hull conv $X$ that the shape functions $\phi_{i}$ corresponding to nodes $x_{i}$ on the interior of the convex domain vanish on the boundary. Furthermore, if a node $x_{i}$ is an extreme point of conv $X$ then the basis functions have the Kronecker-delta property $\phi_{j}\left(\boldsymbol{x}_{i}\right)=\delta_{j i}$. These ideas are illustrated in fig. 1.

The outcome of this is that imposing essential boundary conditions is as simple as the in the FEM, greatly easing the implementation of meshfree methods based on maximum entropy basis functions.

Some other applications of maximum entropy approximants include extension to second-order consistency $[45,46]$, variational optimisation of the support width parameter $\beta$ [47], approximating smooth manifolds (thin shells) on scattered data points [48] and co-rotational elasticity [49].

\subsection{Construction}

We follow the approach of Sukumar and Wright [31] to construct the basis functions, and a brief overview is given here. The maximum-entropy basis 


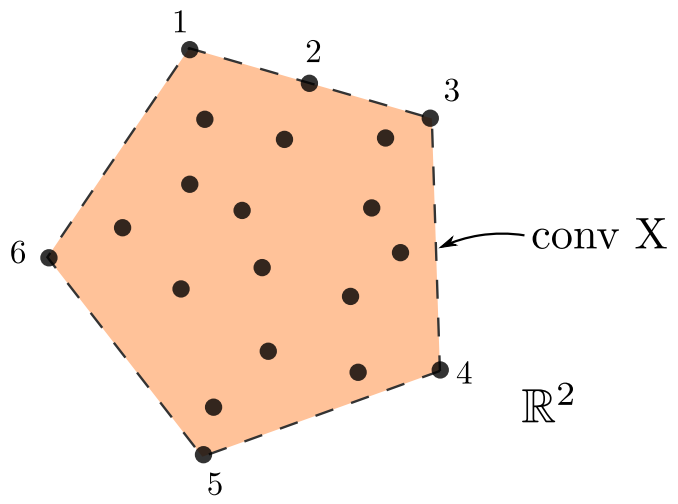

Figure 1: A node set $X$ and its (weakly) convex hull conv $X$ shown by the dashed line. Nodes $\{1,3,4,5,6\}$ have the Kronecker-delta property (interpolatory), whilst node 2 has the 'weak' Kronecker delta property. All internal nodes are non-interpolatory in a similar way to basis functions constructed with MLS.

functions $\phi_{i}$ may be found from the solution of a convex optimisation problem of the form [31]:

$$
\min _{\phi \in \mathbb{R}_{+}^{n}} \sum_{i=1}^{n} \phi_{i}(\boldsymbol{x}) \ln \left(\frac{\phi_{i}(\boldsymbol{x})}{w_{i}(\boldsymbol{x})}\right)
$$

subject to the constraints [31]:

$$
\begin{gathered}
\sum_{i=1}^{n} \phi_{i}(x)=1 \\
\sum_{i=1}^{n} \phi_{i}(x) x_{i}=x \\
\phi_{i}(x) \geq 0 \quad \forall i, x
\end{gathered}
$$

Constraint eq. (5b) is the same as the condition of Partition of Unity and constraint eq. (5d) is required to ensure that the shape functions can be interpreted as probabilities in the context of Shannon's entropy functional. Constraint eq. (5c) ensures that the basis functions can exactly reproduce linear polynomials.

This convex problem is solved numerically using standard techniques from the convex optimisation literature by considering the dual formulation, see eg. Boyd and Vandenberghe [50]. The solution is [31]:

$$
\begin{gathered}
\phi_{i}(\boldsymbol{x})=\frac{Z_{i}(\boldsymbol{x} ; \boldsymbol{\lambda})}{Z(\boldsymbol{x} ; \boldsymbol{\lambda})} \\
Z_{i}(\boldsymbol{x}, \boldsymbol{\lambda})=w_{i}(\boldsymbol{x}) \exp \left(-\boldsymbol{\lambda} \cdot\left(\boldsymbol{x}-\boldsymbol{x}_{i}\right)\right)
\end{gathered}
$$




$$
Z(x ; \lambda)=\sum_{i} Z_{i}(x, \lambda)
$$

where $\lambda=\left[\lambda_{1}, \lambda_{2}, \ldots, \lambda_{d}\right]^{T}$ is a vector of Lagrange multipliers and $d$ is the dimensionality of the domain. The solution for the Lagrange multipliers $\lambda^{*}$ can be found from the following optimisation problem:

$$
\lambda^{*}=\arg \min \ln Z(\lambda)
$$

Denoting $\phi^{*}$ as the basis function solution corresponding to the Lagrange multipliers $\lambda^{*}$ calculated at a particular point $x$ we can then write the basis functions as:

$$
\phi_{i}^{*}(x)=\frac{Z_{i}\left(x ; \lambda^{*}\right)}{Z\left(x ; \lambda^{*}\right)}
$$

and the basis function spatial derivatives as:

$$
\begin{array}{r}
\nabla \phi_{i}^{*}(\boldsymbol{x})=\phi_{i}\left[\left(\boldsymbol{x}-\boldsymbol{x}_{i}\right) \cdot\left(\boldsymbol{H}^{-1}-\boldsymbol{H}^{-1} \cdot \boldsymbol{A}\right)\right. \\
\left.+\frac{\nabla w_{i}}{w_{i}}-\sum_{j=1}^{n} \phi_{j} \frac{\nabla w_{j}}{w_{j}}\right]
\end{array}
$$

where:

$$
\boldsymbol{A}=\sum_{j=1}^{n} \phi_{j}\left(\boldsymbol{x}-\boldsymbol{x}_{i}\right) \otimes \frac{\nabla w_{j}}{w_{j}}
$$

and $\boldsymbol{H}$ is the Hessian matrix calculated with respect to the Lagrange multipliers:

$$
\boldsymbol{H}=\sum_{j=1}^{n} \phi_{j}\left(\boldsymbol{x}-\boldsymbol{x}_{i}\right) \otimes\left(x-x_{i}\right)
$$

The above equations for the shape function derivatives $\nabla \phi_{i}^{*}(x)$ simplify significantly when the Gaussian prior weight function is used [31]:

$$
\nabla \phi_{i}^{*}(\boldsymbol{x})=\phi_{i} \boldsymbol{H}^{-1} \cdot\left(\boldsymbol{x}-\boldsymbol{x}_{i}\right)
$$

\section{Governing equations and mixed formulation}

The derivation of the Reissner-Mindlin plate equations starts with the full 3D equations of linear elasticity which are then simplified by making a set of appropriate geometrical, kinematical and mechanical assumptions [51]. We do not go into great detail, nor attempt to justify the validity of plate models, see eg. [52] for a variational justification. 


\subsection{Strong Form}

Consider an open bounded domain $\Omega \subset \mathbb{R}^{3}$ with boundary $\Gamma$. We define a point $x=\left\{x_{1}, x_{2}, x_{3}\right\} \in \Omega$. We then assume that the domain is thin in the $x_{3}$ direction and that the thickness $t$ is given by a function $t: \Omega_{0} \rightarrow \mathbb{R}$. Therefore the whole domain $\Omega$ can be described by [51]:

$$
\begin{array}{r}
\Omega \equiv\left\{\left(x_{1}, x_{2}, x_{3}\right) \in \mathbb{R}^{3}:\left(x_{1}, x_{2}\right) \in \Omega_{0},\right. \\
\left.x_{3} \in\left[-t\left(x_{1}, x_{2}\right) / 2, t\left(x_{1}, x_{2}\right) / 2\right]\right\}
\end{array}
$$

where the domain $\Omega_{0} \subset \mathbb{R}^{2}$ describes the plate mid-surface. The edges of the plate are then given by [51]:

$$
\begin{array}{r}
\Gamma_{E} \equiv\left\{\left(x_{1}, x_{2}, x_{3}\right) \in \mathbb{R}^{3}:\left(x_{1}, x_{2}\right) \in \Gamma_{0},\right. \\
\left.x_{3} \in\left[-t\left(x_{1}, x_{2}\right) / 2, t\left(x_{1}, x_{2}\right) / 2\right]\right\}
\end{array}
$$

where $\Gamma_{0}$ is the boundary of the domain $\Omega_{0}$. The displacement vector $u: \Omega \rightarrow$ $\mathbb{R}^{3}$ takes the specific form [51]:

$$
\begin{aligned}
\boldsymbol{u}\left(x_{1}, x_{2}, x_{3}\right)=\{ & z_{1}\left(x_{1}, x_{2}\right)-\theta_{1}\left(x_{1}, x_{2}\right) x_{3}, \\
& z_{2}\left(x_{1}, x_{2}\right)-\theta_{2}\left(x_{1}, x_{2}\right) x_{3}, \\
& \left.z_{3}\left(x_{1}, x_{2}\right)\right\}^{T}
\end{aligned}
$$

where $z\left(x_{1}, x_{2}\right)=\left\{z_{1}, z_{2}, z_{3}\right\}^{T}$ describes the displacement of a point on the mid-surface of the plate in the directions $\left(x_{1}, x_{2}, x_{3}\right)$, and $\boldsymbol{\theta}\left(x_{1}, x_{2}\right)=\left\{\theta_{1}, \theta_{2}\right\}^{T}$ describes rotations around the transverse normals. Note that the state of the plate $\boldsymbol{U}=\{\boldsymbol{z}, \boldsymbol{\theta}\}^{T} \in\left(\mathcal{V}_{3}, \mathcal{R}\right)$ is now only a function of the coordinates on the plate mid-surface $\left(x_{1}, x_{2}\right) \in \Omega_{0}$. This allows us to make a trade between integration over the thickness that would be required for the full 3D equations of elasticity for the restricted set of kinematics in (15) [1]. We also assume that the plate is in a state of plane stress [51]:

$$
\sigma_{33}=0
$$

By making the further assumption of isotropic material properties we relate stresses $\sigma_{\alpha \beta}$ to the strains $\epsilon_{\lambda \mu}$ by the reduced constitutive equations in 


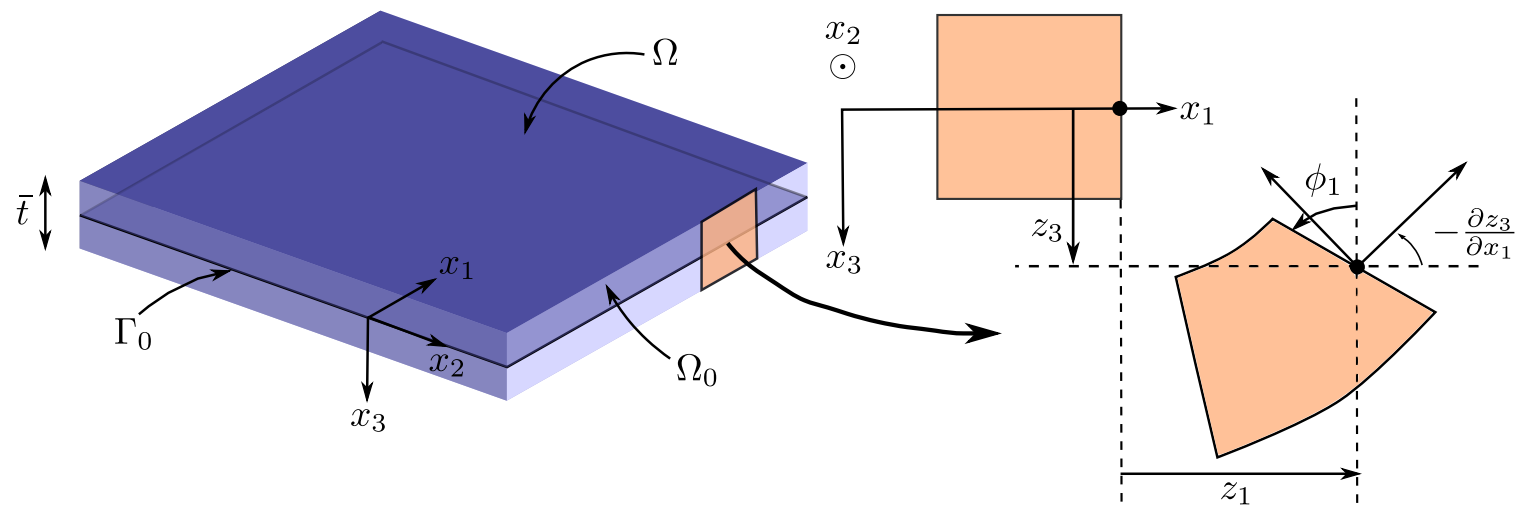

Figure 2: The Reissner-Mindlin plate problem showing displacements $\left(z_{1}, z_{3}\right)$ and rotation $\theta_{1}$.

tensor form [1]:

$$
\begin{gathered}
\sigma_{\alpha \beta}=C_{\alpha \beta \lambda \mu} \epsilon_{\lambda \mu} \\
\sigma_{\alpha 3}=\frac{1}{2} D_{\alpha \lambda} \epsilon_{\lambda 3} \\
C_{\alpha \beta \lambda \mu}=\frac{E}{2(1+v)}\left(\delta_{\alpha \lambda} \delta_{\beta \mu}+\delta_{\alpha \mu} \delta_{\beta \lambda}+\right. \\
\left.\frac{2 v}{1-v} \delta_{\alpha \beta} \delta_{\lambda \mu}\right) \\
D_{\alpha \lambda}=\frac{2 E}{1+v} \delta_{\alpha \lambda}
\end{gathered}
$$

where Greek indices $(\alpha, \beta, \lambda, \mu)$ vary over the set $\{1,2\}, \delta_{\alpha \beta}$ is the usual Kronecker delta function, $E$ is the Young's modulus and $v$ is the Poisson's ratio of the plate material.

After some primarily algebraic work, we can arrive at the strong form of the Reissner-Mindlin equations [1]:

$$
\begin{aligned}
(t \nabla \cdot L \epsilon(\boldsymbol{z}))_{\alpha} & =-p_{\alpha} & & \forall x \in \Omega_{0} \\
\lambda t \nabla \cdot\left(\nabla z_{3}-\boldsymbol{\theta}\right) & =-p_{3} & & \forall x \in \Omega_{0} \\
\left(\frac{t^{3}}{12} \nabla \cdot L \epsilon(\boldsymbol{\theta})+\lambda t\left(\nabla z_{3}-\boldsymbol{\theta}\right)\right) & =0 & & \forall x \in \Omega_{0}
\end{aligned}
$$

where the operator $L$ takes a second-rank tensor $\tau$ :

$$
L[\tau]:=D[(1-v) \tau+v \operatorname{tr}(\tau) I]
$$

and the operator $\epsilon$ is the usual measure of small-strain acting on a vector $v$ :

$$
\epsilon(\boldsymbol{v}):=\frac{1}{2}\left(\nabla \boldsymbol{v}+(\nabla \boldsymbol{v})^{T}\right)
$$


$D$ and $\lambda$ are material constants, relating to the bending and shear behaviour of the material respectively:

$$
D=\frac{E}{1-v^{2}}, \quad \lambda=\frac{E \kappa}{2(1+v)}
$$

$\kappa$ is known as the shear correction factor and accounts for the difference in strain energy created by the assumption of linear shear strains through the thickness and the actual shear strains which must vanish on the top and bottom surfaces of the plate [1].

\subsection{Weak Form}

The strong form of the Reissner-Mindlin problem (18) contains two decoupled sets of equations; the first described by eq. (18a) is known as the in-plane problem as it involves only the in-plane displacements $\left\{z_{1}, z_{2}\right\}^{T}$ and the second described by eqs. (18b) and (18c) are known is known as the out-of-plane problem as it involves only the out-of-plane displacement $z_{3}$ and rotations $\boldsymbol{\theta}$. The solution of the in-plane problem is straightforward. It is the out-of-plane problem that we will concentrate on here as it is this problem in which the difficulty of shear-locking occurs.

Following a standard Bubnov-Galerkin type procedure, we multiply the strong form of the out-of-plane problem eqs. (18b) and (18c) by a set of test functions $y_{3}$ and $\eta$ respectively, integrate over the domain $\Omega_{0}$ and then integrate by parts to derive the equivalent weak form.

We consider trial functions $z_{3} \in \mathcal{V}_{3}$ and $\boldsymbol{\theta} \in \mathcal{R}$ and test functions $y_{3} \in$ $H_{0}^{1}\left(\Omega_{0}\right)$ and $\eta \in\left[H_{0}^{1}\left(\Omega_{0}\right)\right]^{2}$ where $H^{1}\left(\Omega_{0}\right)$ is the usual Sobelov space of square-integrable functions with square-integrable first derivatives in the domain $\Omega_{0}$. Furthermore, $H_{0}^{1}\left(\Omega_{0}\right) \subset H^{1}\left(\Omega_{0}\right)$ is the subset of functions in $H^{1}\left(\Omega_{0}\right)$ with vanishing values (in the sense of traces) on $\Gamma_{0}$. The exact specification of $\mathcal{V}_{3}$ and $\mathcal{R}$ depends on the Dirichlet (displacement) boundary conditions. For example, for hard clamped conditions the spaces are $\mathcal{V}_{3}:=H_{0}^{1}\left(\Omega_{0}\right)$ and $\mathcal{R}:=\left[H_{0}^{1}\left(\Omega_{0}\right)\right]^{2}[53]$.

We normalise the constant thickness $t$ with respect to the characteristic in-plane dimension of the plate $L$ :

$$
\bar{t}=\frac{t}{L}
$$

With this normalisation in place we scale the load by a factor of $\bar{t}^{3}$ such that $p_{3}=g \bar{t}^{3}$ to ensure that the solution $\boldsymbol{U}$ is bounded as $\bar{t} \rightarrow 0$ [1].

The weak form of the out-of-plane problem is: Find $\left(z_{3}, \boldsymbol{\theta}\right) \in \mathcal{V}_{3} \times \mathcal{R}$ such that [1]:

$$
\begin{array}{r}
\int_{\Omega_{0}} L \epsilon(\theta): \epsilon(\eta) d \Omega+\lambda \bar{t}^{-2} \int_{\Omega_{0}}\left(\nabla z_{3}-\boldsymbol{\theta}\right) \cdot\left(\nabla y_{3}-\boldsymbol{\eta}\right) d \Omega \\
=\int_{\Omega_{0}} g y_{3} d \Omega \quad \forall\left(y_{3}, \boldsymbol{\eta}\right) \in \mathcal{V}_{3} \times \mathcal{R}
\end{array}
$$


We then define the scaled transverse shear strain $\gamma=\left(\gamma_{x z}, \gamma_{y z}\right)$ as [51]:

$$
\gamma=\lambda \bar{t}^{-2}\left(\nabla z_{3}-\boldsymbol{\theta}\right) \in \mathcal{S}
$$

allowing us to re-write eq. (23) in equivalent mixed weak form, treating the shear strains $\gamma$ as an independent variational quantity. We introduce strain trial functions $\gamma \in \mathcal{S}$ and strain test functions $\psi \in \mathcal{S}$ to arrive at the following constrained problem [51]: Find $\left(z_{3}, \boldsymbol{\theta}, \gamma\right) \in \mathcal{V}_{3} \times \mathcal{R} \times \mathcal{S}$ such that:

$$
\begin{array}{r}
\int_{\Omega_{0}} L \epsilon(\boldsymbol{\theta}): \epsilon(\boldsymbol{\eta}) d \Omega+\int_{\Omega_{0}} \gamma \cdot\left(\nabla y_{3}-\boldsymbol{\eta}\right) d \Omega \\
=\int_{\Omega_{0}} g y_{3} d \Omega \\
\int_{\Omega_{0}}\left(\nabla z_{3}-\boldsymbol{\theta}\right) \cdot \psi d \Omega-\frac{\bar{t}^{2}}{\lambda} \int_{\Omega_{0}} \gamma \cdot \psi d \Omega=0 \\
\forall\left(y_{3}, \boldsymbol{\eta}, \psi\right) \in \mathcal{V}_{3} \times \mathcal{R} \times \mathcal{S}
\end{array}
$$

The space $\mathcal{S}$ can be characterised as follows [51]. Given $z_{3} \in H_{0}^{1}\left(\Omega_{0}\right)$ it must hold that $\operatorname{rotgrad} z_{3}=0 \in L^{2}\left(\Omega_{0}\right)$. The rot operator ${ }^{1}$ acts on a two component vector field $\boldsymbol{q}=\left(q_{1}, q_{2}\right)$ as [51]:

$$
\operatorname{rot}(\boldsymbol{q})=\frac{\partial q_{2}}{\partial x_{1}}-\frac{\partial q_{1}}{\partial x_{2}}
$$

For $\boldsymbol{\theta} \in\left[H_{0}^{1}\left(\Omega_{0}\right)\right]^{2}$ it holds that $\operatorname{rot} \boldsymbol{\theta} \in L^{2}\left(\Omega_{0}\right)$ and therefore:

$$
\nabla z_{3}-\boldsymbol{\theta} \in H\left(\text { rot; } \Omega_{0}\right)
$$

Thus the shear space $\mathcal{S}$ for fixed $\bar{t}$ can be identified with the space $H\left(\operatorname{rot}, \Omega_{0}\right)$. The space $H\left(\operatorname{rot} ; \Omega_{0}\right)$ is the Sobelov space of square integrable functions with square-integrable rot and is defined as [51]:

$$
H\left(\operatorname{rot}, \Omega_{0}\right):=\left\{\boldsymbol{q} \in\left[L^{2}\left(\Omega_{0}\right)\right]^{2} \mid \operatorname{rot} \boldsymbol{q} \in L^{2}\left(\Omega_{0}\right)\right\}
$$

In the limiting case $\bar{t} \rightarrow 0$ the above equations only hold with the dual space of $\mathcal{S}$ defined by $\mathcal{S}^{\prime}=\left(H\left(\operatorname{rot}, \Omega_{0}\right)\right)^{\prime}=H^{-1}\left(\operatorname{div}, \Omega_{0}\right)$. We refer the reader to Bathe et. al. [55] for an in-depth discussion of these issues.

In summary, by introducing the shear strains as an independent variational quantity in the weak form we have re-cast our original problem which deteriorates for thin-plates into a constrained saddle-point formulation with a penalty term which is robust with the small parameter $\bar{t}$.

\footnotetext{
${ }^{1}$ The rot operator is identical to the curl operator in $\mathbb{R}^{2}$, the latter notation used more frequently in the electro-magnetics literature, see eg. [54]. However, the curl operator extends its definition to $\mathbb{R}^{3}$ so we will use rot here to denote this restriction for our application.
} 


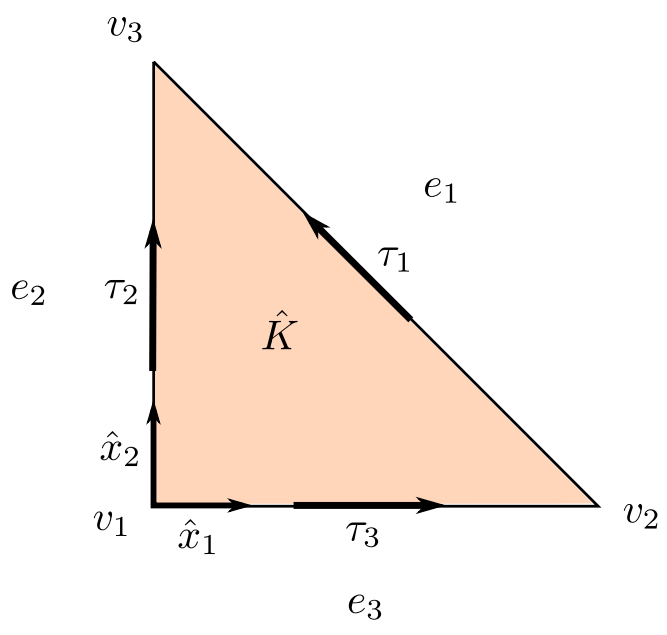

Figure 3: Reference triangle $\hat{K}$ with vertices $v_{i}$ numbered starting anti-clockwise from $v_{0}=(0,0)$. Edges $e_{i}$ are numbered corresponding to the opposite vertex. Edge tangent vectors $\tau_{i}$ are shown.

\subsection{Discrete Weak Form}

\subsubsection{Mixed discrete weak form}

We begin by discretising the shear strains $\gamma$. To build a conforming subspace of $H\left(\right.$ rot; $\left.\Omega_{0}\right)$ we use rotated Raviart-Thomas-Nédélec [32, 33] elements of lowest order on a triangular background mesh. These elements are often referred to as edge elements as their degrees of freedom are defined as integrals along the element edges. We refer to this family of elements as $\mathrm{NED}_{q}$ for $q=1,2, \ldots$, where $q$ refers to the order of polynomial included in the ba$\mathrm{sis}^{2}$. These elements can be viewed as rotated versions of those introduced by Raviart and Thomas [32] to build conforming subspaces of $H($ div; $\Omega$ ) as the rot and div operator can be related by a rotation of a two component vector field $q$ by $\pi / 2$.

We denote the discrete solution for the shear strains as $\gamma_{h}(x) \in \mathcal{S}_{h}$ where $\mathcal{S}_{h}:=\operatorname{NED}_{1}\left(\Omega_{0} ; \mathcal{T}_{h}\right) \subset H\left(\operatorname{rot} ; \Omega_{0}\right)$. $\mathcal{T}_{h}$ is a triangulation on $\Omega_{0}$ with edges $\mathcal{E}_{h}$. For a reference triangle $\hat{K}$ with edges $e \in \mathcal{E}_{h}(\hat{K})$ (see fig. 3 ) and degrees of freedom $\Sigma_{i}$ defined on the edges $e_{i}$ the interpolation across $\hat{K}$ can be written

\footnotetext{
${ }^{2}$ In Raviart and Thomas's original paper [32] on constructing conforming subspaces of $H(\operatorname{div} ; \Omega)$ they numbered their elements starting with $q=0$, so the lowest-order element is called $R T_{0}$. In this paper we use the convention of Nédélec [33] and start with $q=1$ as the final polynomial space for the element includes terms of order $\mathbb{P}_{q}(\hat{K})$
} 


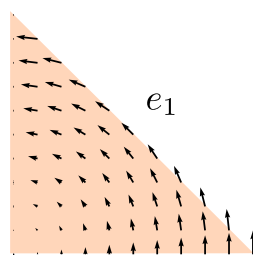

$N_{1}$

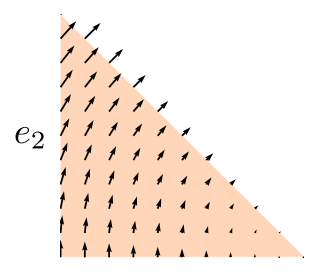

$N_{2}$

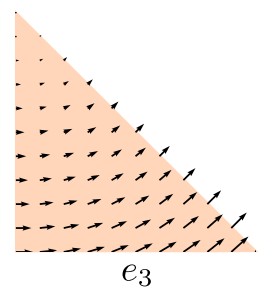

$N_{3}$

Figure 4: Basis functions $N_{i}$ associated with edge $e_{i}$ on the reference triangle $\hat{K}$.

as:

$$
\begin{aligned}
\gamma_{h}\left(\hat{x}_{1}, \hat{x}_{2}\right) & =\sum_{i=1}^{3} N_{i} \gamma_{i} \\
& =\left[\left(\begin{array}{c}
-\hat{x}_{2} \\
\hat{x}_{1}
\end{array}\right)\left(\begin{array}{c}
\hat{x}_{2} \\
1-\hat{x}_{1}
\end{array}\right)\left(\begin{array}{c}
1-\hat{x}_{2} \\
\hat{x}_{1}
\end{array}\right)\right]\left\{\begin{array}{l}
\gamma_{1} \\
\gamma_{2} \\
\gamma_{3}
\end{array}\right\} \\
& =N_{\gamma} \gamma \quad \forall\left(\hat{x}_{1}, \hat{x}_{2}\right) \in \hat{K}
\end{aligned}
$$

where $\left(\hat{x}_{1}, \hat{x}_{2}\right)$ is the coordinate system in the reference triangle $\hat{K}$. An overview of the construction of these shape functions is given in Appendix A and the transformation to a general element $K$ is given in Appendix B. Similarly, we denote the discrete solution for the tranverse displacement and rotations as $z_{3 h} \in \mathcal{V}_{3 h}$ and $\boldsymbol{\theta}_{h} \in \mathcal{R}_{h}$ where $\mathcal{V}_{3 h}=\mathcal{R}_{h}:=\operatorname{ME}\left(\Omega_{0} ; \mathcal{N}, \rho\right) \subset H_{0}^{1}\left(\Omega_{0}\right) . \mathcal{N}_{h}$ is a set of nodes in $\Omega_{0}$ associated with a set of support sizes $\rho$. Here we assume that $\Omega_{0}$ is a convex domain so that all basis functions $\phi_{i}$ associated with nodes inside the domain vanish on the boundary. We can then write the trial functions for the displacement and rotations as:

$$
\begin{aligned}
z_{3 h}(x) & =\sum_{i=1}^{N} \phi_{i} z_{3 i}=\left[\begin{array}{llll}
\phi_{1} & \phi_{2} & \ldots & \phi_{N}
\end{array}\right]\left\{\begin{array}{c}
z_{31} \\
z_{32} \\
\vdots \\
z_{3 N}
\end{array}\right\} \\
& =\boldsymbol{\phi}_{z_{3}} z_{3} \quad \forall x \in \Omega_{0}
\end{aligned}
$$




$$
\begin{aligned}
\boldsymbol{\theta}_{h}(\boldsymbol{x}) & =\sum_{i=1}^{N} \boldsymbol{\phi}_{i} \boldsymbol{\theta}_{i} \\
& =\left[\begin{array}{cccccccc}
\phi_{1} & \phi_{2} & \ldots & \phi_{N} & 0 & 0 & \ldots & 0 \\
0 & 0 & \ldots & 0 & \phi_{1} & \phi_{2} & \ldots & \phi_{N}
\end{array}\right]\left\{\begin{array}{c}
\theta_{x 1} \\
\theta_{x 2} \\
\vdots \\
\theta_{x N} \\
\theta_{y 1} \\
\theta_{y 2} \\
\vdots \\
\theta_{y N}
\end{array}\right\} \\
& =\Phi_{\theta} \boldsymbol{\theta} \quad \forall \boldsymbol{x} \in \Omega_{0}
\end{aligned}
$$

We define our trial functions using the same basis as the test functions:

$$
y_{3 h}=\boldsymbol{\phi}_{z 3}, \quad \eta_{h}=\boldsymbol{\Phi}_{\boldsymbol{\theta}}, \quad \boldsymbol{\psi}_{h}=\boldsymbol{N}_{\gamma}
$$

We can then write the discrete linear set of equations as:

$$
\begin{gathered}
\int_{\Omega_{0}} \boldsymbol{B}_{b}^{T} \boldsymbol{D}_{b} \boldsymbol{B}_{b} d \Omega \boldsymbol{\theta}+\int_{\Omega_{0}} \boldsymbol{B}_{s}^{T} \boldsymbol{N}_{\gamma} d \Omega \gamma=\int_{\Omega_{0}} \boldsymbol{\phi}_{z 3} g d \Omega \\
\int_{\Omega_{0}} \boldsymbol{N}_{\gamma}^{T} \boldsymbol{B}_{s} d \Omega\left\{\begin{array}{c}
\boldsymbol{\theta} \\
z_{3}
\end{array}\right\}-\bar{t}^{2} \boldsymbol{D}_{s}^{-1} \int_{\Omega_{0}} \boldsymbol{N}_{\gamma}^{T} \boldsymbol{N}_{\gamma} d \Omega \gamma=0
\end{gathered}
$$

where the $\boldsymbol{B}_{b} \in \mathbb{R}^{3 \times 3 N}$ and $\boldsymbol{B}_{s} \in \mathbb{R}^{2 \times 3 N}$ are matrices containing componentwise derivatives of the shape function vectors:

$$
\begin{gathered}
\boldsymbol{B}_{b}=\left[\begin{array}{cc}
\frac{\partial \phi_{\theta}}{\partial x_{1}} & \mathbf{0} \\
\mathbf{0} & \frac{\partial \phi_{\theta}}{\partial x_{2}} \\
\frac{\partial \phi_{\theta}}{\partial x_{2}} & \frac{\partial \phi_{\theta}}{\partial x_{1}}
\end{array}\right] \\
\boldsymbol{B}_{s}=\left[\begin{array}{ccc}
-\boldsymbol{\phi}_{\theta_{x}} & 0 & \frac{\partial \phi_{z 3}}{\partial x_{1}} \\
0 & -\boldsymbol{\phi}_{\theta_{y}} & \frac{\partial \phi_{z 3}}{\partial x_{2}}
\end{array}\right]
\end{gathered}
$$

and $\boldsymbol{D}_{s} \in \mathbb{R}^{2 \times 2}$ and $\boldsymbol{D}_{b} \in \mathbb{R}^{3 \times 3}$ are matrices containing the material properties of the plate:

$$
\begin{gathered}
\boldsymbol{D}_{s}=\left[\begin{array}{ll}
\lambda & 0 \\
0 & \lambda
\end{array}\right] \\
\boldsymbol{D}_{b}=D\left[\begin{array}{ccc}
1 & v & 0 \\
v & 1 & 0 \\
0 & 0 & \frac{1-v}{2}
\end{array}\right]
\end{gathered}
$$


The above set of equations is a linear system of the following form:

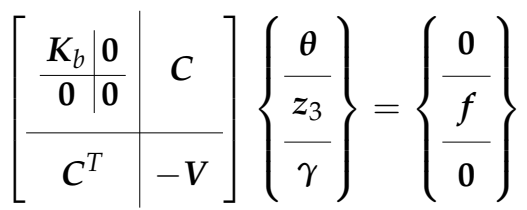

where

$$
\begin{gathered}
\boldsymbol{K}_{b}=\int_{\Omega_{0}} \boldsymbol{B}_{b} \boldsymbol{D}_{b} \boldsymbol{B}_{b} d \Omega \\
\boldsymbol{C}=\int_{\Omega_{0}} \boldsymbol{B}_{s}^{T} \boldsymbol{N}_{\gamma} d \Omega \\
\boldsymbol{V}=\int_{\Omega_{0}} \boldsymbol{N}_{\gamma}^{T} \boldsymbol{N}_{\gamma} d \Omega \\
f=\int_{\Omega_{0}} \boldsymbol{\phi}_{z 3} g d \Omega
\end{gathered}
$$

where $\boldsymbol{K}_{b} \in \mathbb{R}^{2 N \times 2 N}, \boldsymbol{C} \in \mathbb{R}^{3 N \times\left|\mathcal{E}_{h}\right|}, \boldsymbol{V} \in \mathbb{R}^{\left|\mathcal{E}_{h}\right| \times\left|\mathcal{E}_{h}\right|}$ and $\boldsymbol{f} \in \mathbb{R}^{N \times 1}$ where $\left|\mathcal{E}_{h}\right|$ is the number of edges in the triangulation $\mathcal{T}_{h}$ and $N$ is the number of nodes in the node set $\mathcal{N}_{h}$. The total solution vector sizes is of size $3 N+\left|\mathcal{E}_{h}\right|$.

\section{Results}

\subsection{Implementation details}

The method outlined in section 3 was implemented using a combination of Python, $\mathrm{C}++$ and Fortran 90 programming languages. We used a slightly modified version of N. Sukumar's Fortran 90 code [56] to generate the maximum-entropy basis functions. This code was interfaced to our objectoriented $\mathrm{C}++$ library which provides a consistent programming interface for generating a variety of meshless basis functions including MLS and the Radial Point Interpolation Method (RPIM) [57]. We wrapped the C++ library using the Boost Python library [58] to allow direct access from Python. The integration and assembly steps of the numerical method were then implemented in Python using the NumPy [59] library. We used the C++ mesh implementation from the DOLFIN [60] and the FIAT library [61] to tabulate the basis NED . $^{\text {. }}$ We used the ckdtree library from SciPy [62] for accelerated nearest-neighbour searching.

\subsection{Methods used for comparison}

FE 1 Displacement We use standard linear $C^{0}$ continuous Lagrangian elements, denoted $\mathrm{CG}_{1}$, for all fields $\left(\boldsymbol{\theta}, z_{3}\right)$ in a displacements-only weak form. This formulation is prone to shear-locking. 

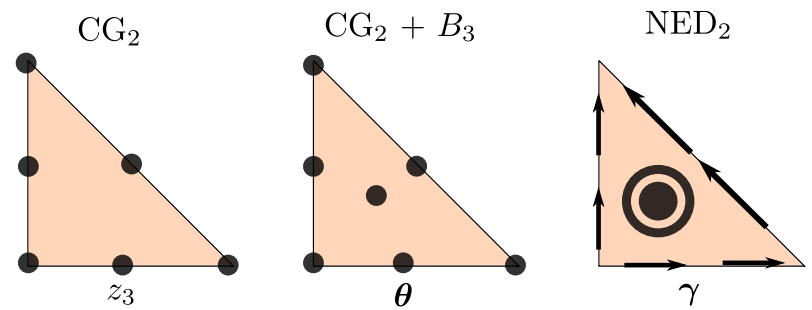

Figure 5: FE 2 Mixed Element Structure; transverse deflections are approximated using continuous second-order Lagrangian element $\mathrm{CG}_{2}$ whilst the rotations are approximated using CG2 enriched with third-order bubble functions $B_{3}$. The shear strains are approximated using $\mathrm{NED}_{2}$ elements which have two internal 'moment' degrees of freedom in addition to two degrees of freedom on each edge.

FE 2 Displacement We use standard quadratic $C^{0}$ continuous Lagrangian elements, denoted $\mathrm{CG}_{2}$, for all fields $\left(\boldsymbol{\theta}, z_{3}\right)$ in a displacements-only weak form. This formulation is prone to shear-locking.

FE 2 Mixed We use the element structure shown in fig. 5 in a mixed weak form.

Maximum-Entropy (MaxEnt) Mixed This is the method outlined in section 3.

\subsection{Parameters}

We define the following parameters as quantities of interest in the construction of both $\mathcal{N}_{h}$ and $\mathcal{T}_{h}$.

On node set $\mathcal{N}_{h}$ we define $h_{a}$ as the distance between node $a$ and its nearest neighbouring node. On a regular grid the distance $h_{a}=h \forall a$ will be the same for all nodes. We then define the support $\rho_{a}$ of node $a$ by the formula:

$$
\rho_{a}=\kappa \gamma h_{a}
$$

On uniform grids of nodes we will take values $\gamma=\sqrt{2}, 2, \sqrt{4+1}, 3$ and $\kappa=1.05$ in an attempt to find a roughly optimal value.

We define the constraint ratio $r$ as the ratio of the number of degrees of freedom in the displacement variable function spaces $\left(\mathcal{V}_{h}, \mathcal{R}_{h}\right)$ to the number of degrees of freedom in the shear strain function space $\mathcal{S}_{h}$ :

$$
r=\frac{\operatorname{dim} \mathcal{V}_{h}+\operatorname{dim} \mathcal{R}_{h}}{\operatorname{dim} \mathcal{S}_{h}}
$$

This quantity is of interest because it gives a rough measure of the relative sizes of the spaces which is inherently related to the stability and performance of the final linear system of equations. We will show through numerical experiments that if the ratio $r$ is too low the method becomes over-constrained and solution quality decreases, and if the ratio $r$ is too high the method becomes under-constrained and unstable. 
(a)

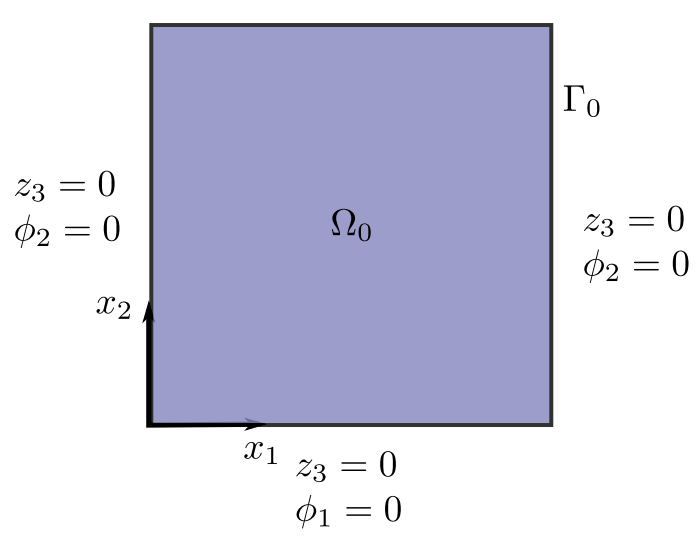

(b)

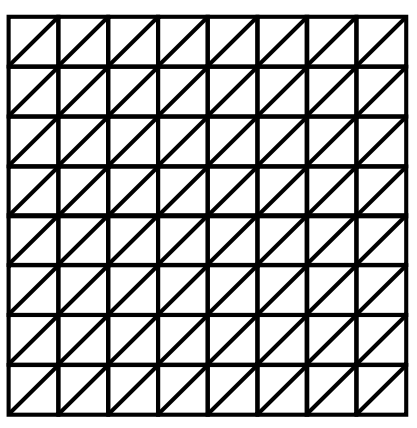

$\mathcal{T}_{h}, N=8$

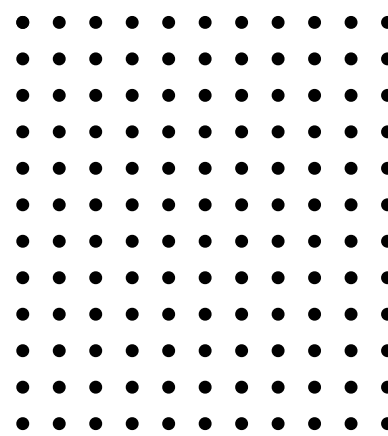

$\mathcal{N}_{h}, M=12$

Figure 6: (a) Domain $\Omega_{0}$ for the SSSS square plate showing boundary conditions on each edge. (b) Example discretisation of square domain.

\subsection{Simply Supported Square Plate with Uniform Pressure}

We use the common setup of a simply supported square plate with uniform pressure as a test problem as it has a closed-form analytical solution [63]. The problem domain $\Omega_{0}$ is defined by:

$$
\Omega_{0}=\left\{\left(x_{1}, x_{2}\right) \in \mathbb{R}^{2}: 0<x_{1}<1,0<x_{2}<1\right\}
$$

and the boundary conditions are hard simply supported:

$$
\boldsymbol{\theta} \cdot \boldsymbol{\tau}=\boldsymbol{n} \cdot \operatorname{L\epsilon }(\boldsymbol{\theta}) \boldsymbol{n}=z_{3}=0 \quad \forall \boldsymbol{x} \in \Gamma_{0}
$$

where $\boldsymbol{n}$ is the unit normal vector to the boundary and $\tau$ is the unit tangent vector to the boundary. The SSSS domain and boundary conditions are shown in ??.

We take the following numerical values: $E=10920, v=0.3, \kappa=5 / 6, g=$ 1 and define the following normalised transverse displacement $\hat{z}_{3}$ [63]:

$$
\hat{z}_{3}=\frac{E}{12\left(1-v^{2}\right)} z_{3} \times 10^{2}=z_{3} \times 10^{5}
$$

Note that there is no factor of $\bar{t}^{3}$ as in Reddy [63] as we have already scaled the loading $f$ by a factor of $g \bar{t}^{3}$ in the governing weak form to ensure the solution is bounded as $\bar{t} \rightarrow 0$.

We define the $L^{2}$ relative error in $e_{L^{2}}\left(u_{h}, u\right)$ between the numerical solution 


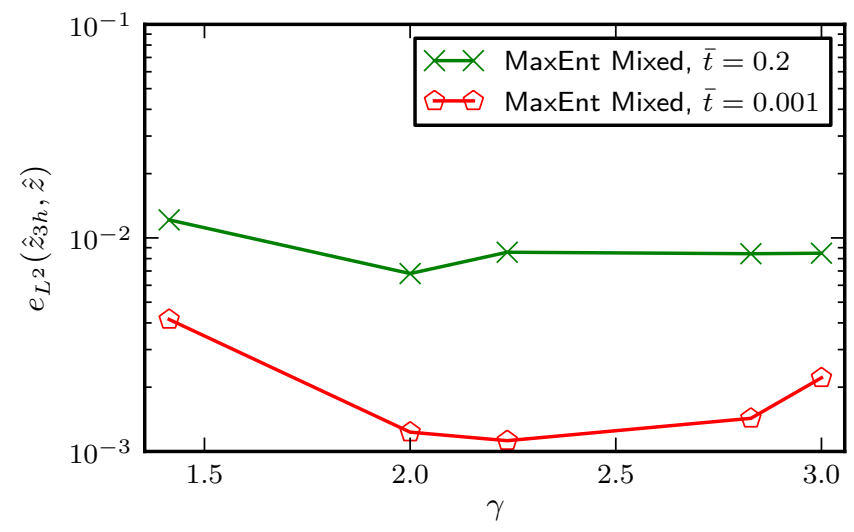

Figure 7: Graph showing the effect of the parameter $\gamma$ on convergence. $N=8, M=12$.

$u_{h}$ and exact solution $u$ as:

$$
e_{L^{2}}\left(u_{h}, u\right)=\frac{\left\|u-u_{h}\right\|_{L^{2}(\Omega)}}{\|u\|_{L^{2}(\Omega)}}=\frac{\left(\int_{\Omega_{0}}\left(u-u_{h}\right)^{2} d \Omega\right)^{1 / 2}}{\left(\int_{\Omega_{0}} u^{2} d \Omega\right)^{1 / 2}}
$$

In the case of the simply supported plate where we have an analytical solution available we will use the above as an indicator of solution quality.

\subsubsection{Parameters}

In fig. 7 we show the effect of the parameter $\gamma$ on the error in the $L^{2}$ norm for thick $\bar{t}=0.2$ and thin $\bar{t}=0.001$ plates. We can see that a value of $\gamma=\sqrt{2}$ is insufficient and that values of $\gamma \geq 2$ appear to be optimal. For $\gamma \geq \sqrt{4+1}$ there seems to a slight increase in error for the thick plate, whilst error is minimised at $\gamma=\sqrt{4+1}$ for the thin plate. However these variations are small enough that we take a value of $\gamma=2$ to minimise the bandwidth of the linear system as well as shape function computation time.

We discretise the domain as shown in ??, using a uniform triangulation $\mathcal{T}_{h}$ with $N$ cells along an edge, and a uniform node set $\mathcal{N}_{h}$ with $M$ nodes along an edge.

In fig. 8 we demonstrate the effect of the constraint ratio $r$ on the error in the $L^{2}$ norm for varying $\bar{t}$. We use a fixed maximum-entropy node set $\mathcal{N}_{h}$ with $M=12$ and vary the underlying uniform triangulation $\mathcal{T}_{h}$ by adjusting $N$ to achieve combinations of $\left(\mathcal{N}_{h}, \mathcal{T}_{h}\right)$ with varying constraint ratios $r$.

We note that for thick plates $t \geq 10^{-1}$ the combinations of $\left(\mathcal{N}_{h}, \mathcal{T}_{h}\right)$ with the lowest $r$ value achieve the lowest error, whilst those with the highest $r$ values have the highest error. However, it is clear that the differences in error for thick plates for varying $r$ are small compared to those when the plate is thin $\bar{t} \leq 10^{-2}$ thus we make decisions on the optimal values of $r$ based on 


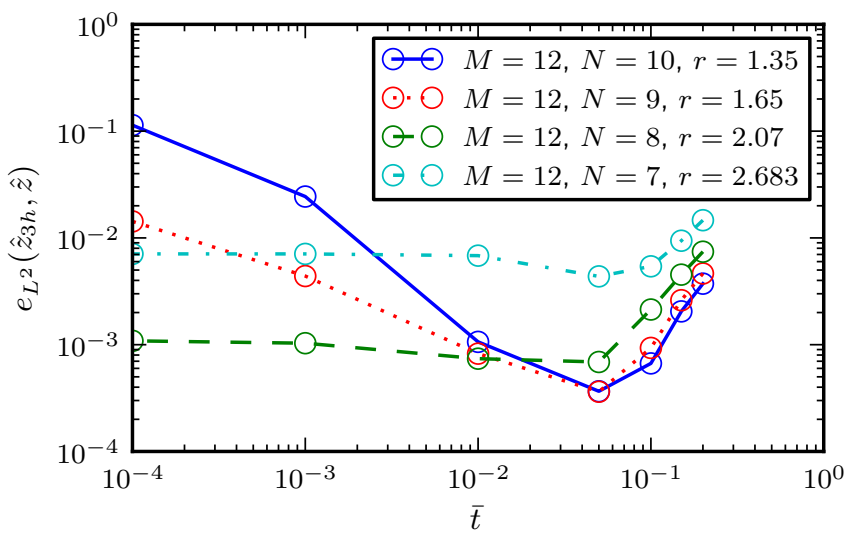

Figure 8: Showing the effect of the constraint ratio $r$ on the solution for varying $\bar{t} . \gamma=2$.

results for thin plates alone. We can see that for $N=9,10$ corresponding to $r=1.35,1.65$ the error increases rapidly as $\bar{t}$ decreases. However, for $N=8$, corresponding to $r=2.07$, the error stays bounded at around $10^{-3}$. For $N=7$, corresponding to $r=2.683$ we have uniformly worse convergence across the entire range of $\bar{t}$. Furthermore to the results shown in fig. 8, we found that the method became unstable for $N \leq 6$ corresponding to values of $r \geq 3.6$ and for $N \geq 10$ we found increasingly poor convergence performance. For the discretisations used in our convergence studies we have found that constraint ratios between 2.0 and 2.5 to be satisfactory.

Because of the non-polynomial nature of the maximum-entropy shape functions accurate integration of the weak form typically requires Gauss quadrature rules of higher order than those used in the FEM. To ensure that we are integrating the weak form with sufficient accuracy we examined the effect of Gauss quadrature order on the error in the $L^{2}$ norm. In fig. 9 we show that Gauss quadrature of order 3 or greater is sufficient to integrate the weak form. In our results we used Gauss quadrature of order 5 to ensure no adverse effects due to integration errors.

\subsubsection{Locking}

In fig. 10 we demonstrate the shear-locking-free property of the proposed method through the convergence of the centre point transverse deflection $\hat{z}_{3 h}(0.5,0.5)$ to the Kirchoff thin plate solution. We show both the Kirchoff and Reissner-Mindlin analytical solutions; for thin plates $10^{-4} \leq \bar{t} \leq 10^{-2}$ the two almost coincide, whilst for thicker plates $t \geq 10^{-2}$ they diverge as the Reissner-Mindlin theory's relaxation upon the rotation of the transverse normals $\nabla z_{3} \neq \theta$ becomes increasingly important. The FE 2 Displacement result clearly shows the pitfalls of using an unmodified displacements-based formulation with severe shear-locking for values of $\bar{t} \leq 10^{-2}$. We note that shear-locking would also occur using the maximum-entropy basis functions, 


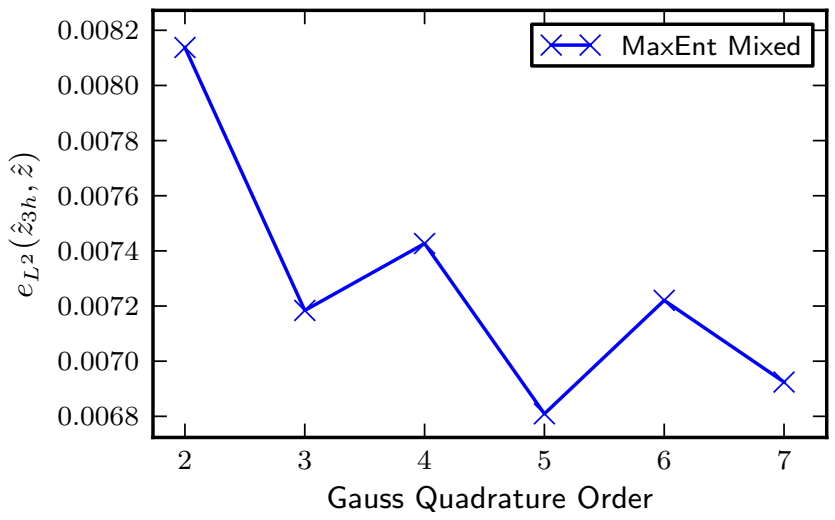

Figure 9: Graph showing the effect of the order of the Gauss quadrature rule used for integration on convergence. $N=8, M=12, \gamma=2.0$.

or indeed any other type of standard basis functions using an unmodified displacements-based weak form. Clearly the proposed maximum-entropy mixed method matches, as desired, the analytical solution throughout the full range of thick and thin plates.

In fig. 11 we show the convergence of $\hat{z}_{3 h}$ to the analytical solution $\hat{z}_{3}$ in the $L^{2}$-norm for varying $\bar{t}$. The error for the proposed maximum-entropy mixed method stays bounded below $10^{-2}$ as $\bar{t} \rightarrow 0$ whilst for the FE 1 displacement method error increases rapidly for $t<10^{-1}$ as the formulation locks. Using the FE 2 mixed formulation also results in a locking-free results.

In fig. 12 we show the convergence of $z_{3 h}$ to the analytical solution $\hat{z}_{3}$ in the $L^{2}$-norm against number of degrees of freedom for various shear-locking and shear-locking-free methods for a thick plate $\bar{t}=0.2$. We note that all of the methods converge in the $L^{2}$-norm. The FE 2 mixed, maximum-entropy mixed and maximum-entropy displacement formulations provide significantly lower errors than the FE 1 mixed and FE 1 displacement methods. The FE 2 mixed formulation has the highest rates of convergence at $\mathcal{O}\left(\operatorname{dof}^{-3 / 2}\right) \sim \mathcal{O}\left(h^{3}\right)$, consistent with quadratic interpolation. The FE 1 displacement, maximumentropy mixed and maximum-entropy displacement formulations have convergence rates of $\mathcal{O}\left(\operatorname{dof}^{-1}\right) \sim \mathcal{O}\left(h^{2}\right)$, consistent with linear interpolation/approximation. We note that comparing the purely meshfree approach vs. our hybrid FE/meshfree mixed approach that both convergence rates and errors are marginally worse for the latter. However, our approach still seems to be competitive with the FE 2 mixed method and significantly better than FE 1 displacement method.

In fig. 13 we show the convergence of $z_{3 h}$ to the analytical solution $\hat{z}_{3}$ in the $L^{2}$-norm against number of degrees of freedom for the proposed maximumentropy mixed and FE 2 mixed shear-locking-free methods for a thin plate $\bar{t}=0.001$. The maximum-entropy mixed approach has a convergence rate of $\mathcal{O}\left(\right.$ dof $\left.^{-1}\right) \sim \mathcal{O}\left(h^{2}\right)$ whilst the FE 2 mixed approach has a convergence rate 


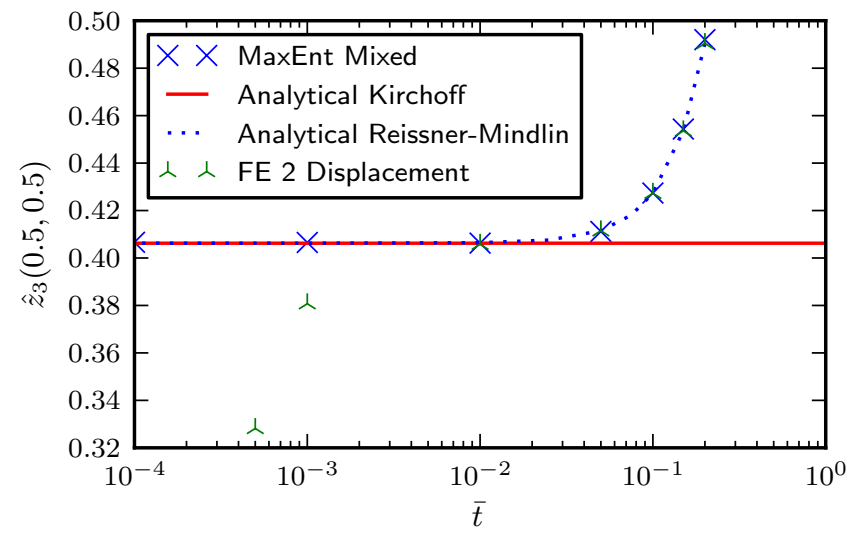

Figure 10: Graph showing normalised central deflection $z_{3}(0.5,0.5)$ of SSSS square plate for varying $\bar{t}$.

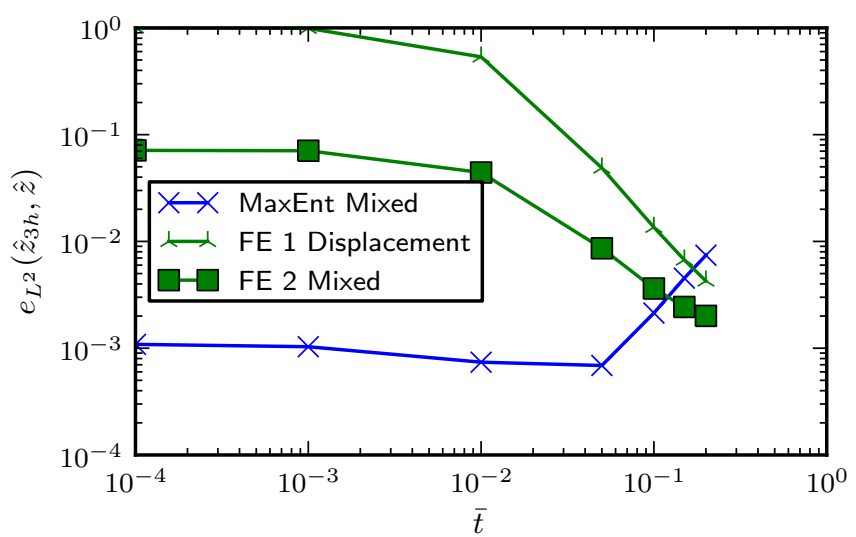

Figure 11: Graph showing error in $\hat{z}_{3 h}$ for varying $\bar{t}$. Maximum-entropy mixed: $N=8, M=$ 12, $\gamma=2$.0. FE 1 displacement: $N=30$. FE 2 mixed $N=8$. 


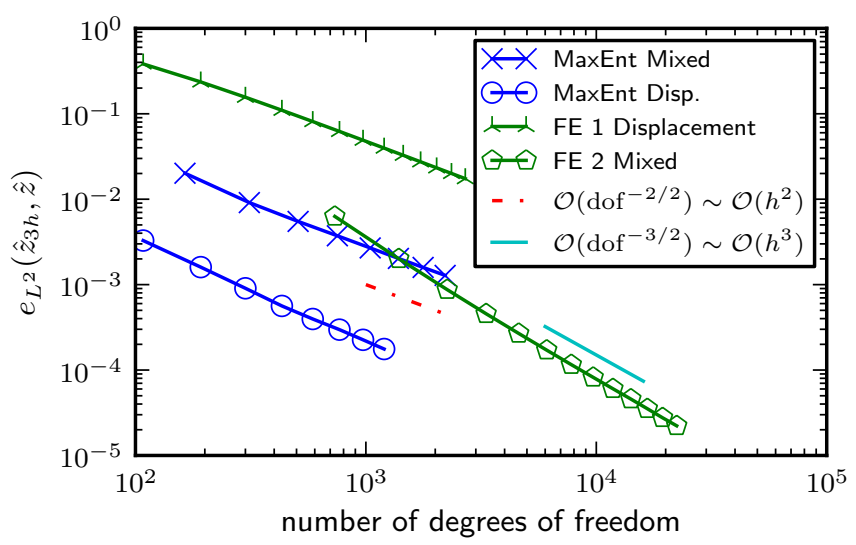

Figure 12: Graph showing $L^{2}$ error in $\hat{z}_{3 h}$ against number of degrees of freedom using various shear-locking and shear-locking-free methods for a thick plate $\bar{t}=0.2$.

of $\mathcal{O}\left(\right.$ dof $\left.^{-3 / 2}\right) \sim \mathcal{O}\left(h^{3}\right)$. For thin plates the proposed method appears to be superior to the FE 2 mixed formulation.

\subsection{Fully Clamped Square Plate with Uniform Pressure}

Using the same domain $\bar{\Omega}_{0}$ as defined in eq. (40) we now apply fully clamped boundary conditions:

$$
\boldsymbol{\theta}=z_{3}=0 \quad \forall x \in \Gamma_{0}
$$

Due to the lack of analytical solution we compute a reference solution on a highly refined mesh $\mathcal{T}_{h}$ with $N=70$ using the FE 2 mixed formulation. We take the Kirchoff centre point deflection as $\hat{z}_{3}=0.126401$ according to İmrak et. al. [64]. Our reference FE Reissner-Mindlin solution agrees with this value to 4 decimal places.

In fig. 14 we show the central deflection of the plate for the proposed method and the FE 2 mixed method alongside the reference solutions. We can see that the proposed method provides competitive results with the FE 2 mixed method.

In fig. 15 we show contour plots of both $\hat{z}_{3 h}$ and $\theta_{1 h}$ with $\bar{t}=0.01$. These contour plots were created by using the maximum-entropy basis functions to approximate the results on a regular grid of points between nodes. Smooth results are easily obtained with no post-processing required.

\section{Concluding remarks}

In this paper we have proposed a method for the locking-free simulation of Reissner-Mindlin plates using a novel combination of maximum entropy basis functions and rotated Raviart-Thomas-Nédélec elements. 


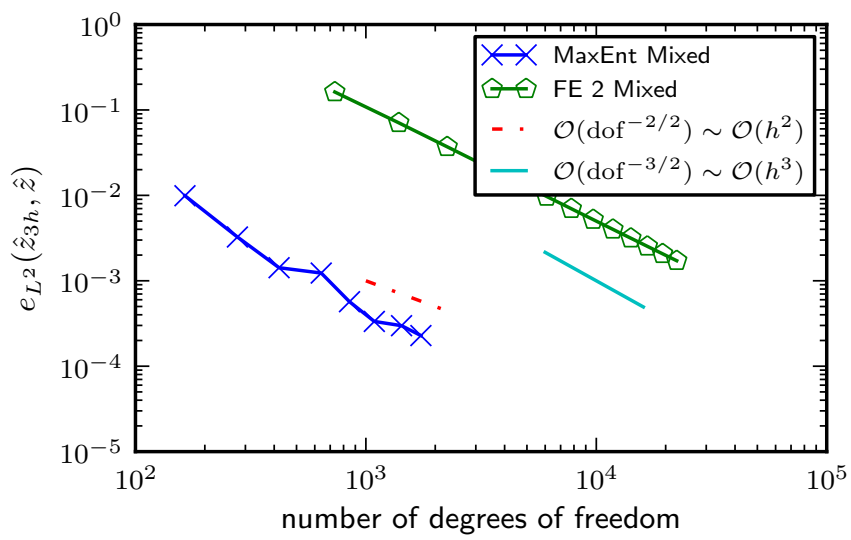

Figure 13: Graph showing $L^{2}$ error in $\hat{z}_{3 h}$ using two locking-free methods for a thin plate $\bar{t}=0.001$.

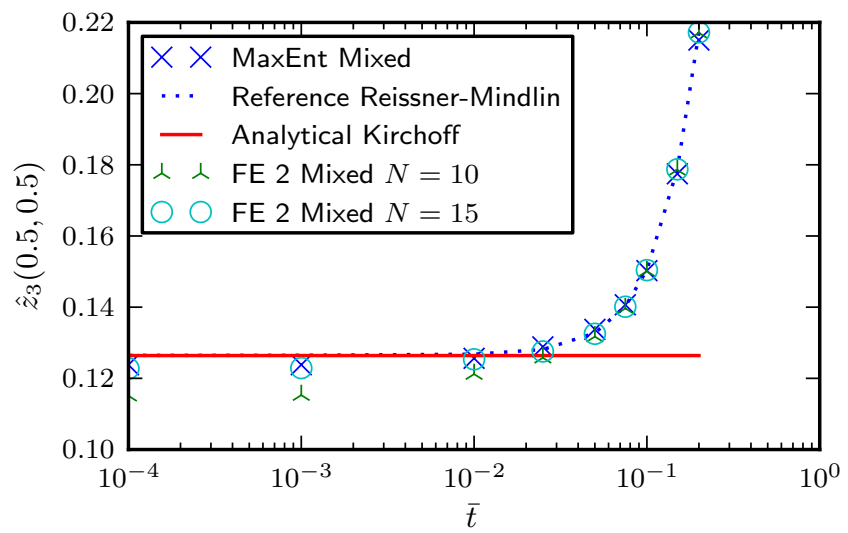

Figure 14: Graph showing normalised central deflection $z_{3}(0.5,0.5)$ of CCCC square plate for varying $\bar{t}$. Maximum-entropy mixed: $N=10, M=16$, dofs $=1088$. FE 2 mixed $N=10$, dofs $=2253$. FE 2 mixed $N=15$, dofs $=5323$. 
(a)

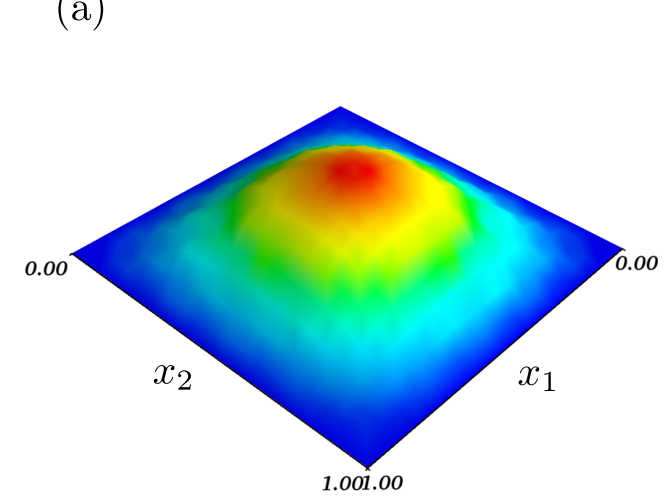

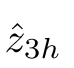

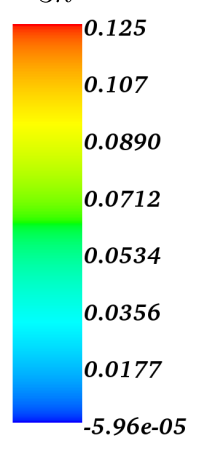

(b)

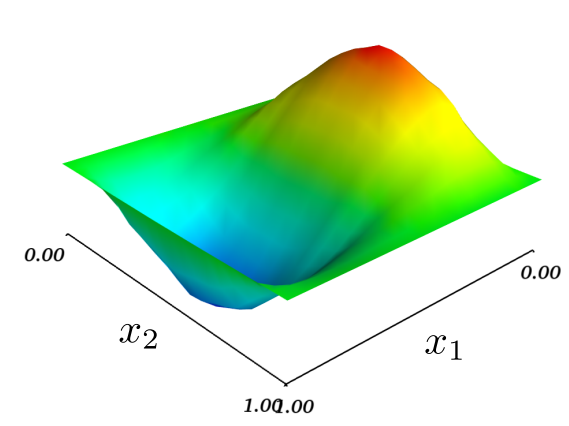

$\theta_{1 h}$

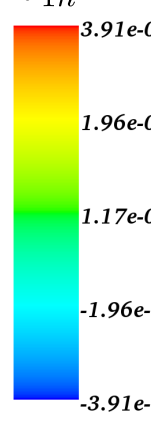

Figure 15: CCCC square plate problem with $\bar{t}=0.01$. (a) Transverse deflection $\hat{z}_{3 h}$. (b) Rotation $\theta_{1 h}$

The use of maximum entropy basis functions has allowed simple and direct imposition of Dirichlet boundary conditions. This 'weak' Kronecker-delta property is an inherent property of the maximum entropy approach, and we believe this affords significant advantages over the more commonly used moving-least-squares basis functions.

We comment that our approach has only required first-order consistency in the meshfree basis functions, whereas approaches from other authors have typically required second-order or higher consistency. This reduces the support size of the basis functions and creates a stiffness matrix with lower bandwidth.

Through some simple test problems we have shown the efficacy of the proposed method which seems to be competitive with the quadratic FEM.

The approach of using a mixed variational form, although well established in the FEM, has not to our knowledge been used to solve the problem of shear-locking in the mesh-free plate literature.

Although we have had to use an underlying mesh for the generation of a $H\left(\operatorname{rot} ; \Omega_{0}\right)$ conforming field, we believe that this is a technological gap between meshfree and FE that is already partially closed, thus opening up the possibility of making our method 'truly' meshfree. We support this assertion by referring to a paper by Buffa et. al. [65] where $H($ curl, $\Omega)$ conforming B-spline shape functions are constructed for the solution of isogeometric electro-magnetics problems.

Again, mimicking the approach used in many successful FE approaches such as the MITC family of elements, the elimination of the shear-strain field $\gamma$ via some kind of projection or reduction operator (typically denoted $\Pi_{h}$ or $R_{h}$ respectively in the literature) may also be possible. We support this by referring to papers by Sukumar et. al. and Ortiz et. al. [28, 29] where, although not explicitly referred to as such, a form of projection operator is 
defined by volume-averaging the pressure field over elements attached to each node, eliminating the pressure field from the final stiffness matrix.

\section{Appendix A. Rotated Raviart-Thomas-Nédélec Elements}

This section gives a brief overview of the construction of the lowest-order rotated Raviart-Thomas-Nédélec elements, here denoted $\mathrm{NED}_{1}$. Whilst these elements are well known in the mathematics literature they seem to be less well known in the engineering community.

We begin with the classical Ciarlet triple definition of a finite element $(T, \mathcal{V}, \mathcal{L})$ where $T$ is an element geometry (triangle, tetrahedron, quadrilateral etc.), $\mathcal{V}$ is a function space (typically a polynomial) defined on $T$, and $\mathcal{L}=\left\{l_{1}, l_{2}, \ldots, l_{n}\right\}$ is a set of degree of freedom which are a set of linear functions on $\mathcal{V}$ [66]. The degrees of freedom $l_{i}$ must be linearly independent.

First of all we will define some polynomial spaces that will be used to define the space $\mathcal{V}$ for our element $\operatorname{NED}_{1}$. We denote $\mathbb{P}_{k}(\Sigma)$ as the set of polynomials of degree $k$ on $\Sigma$, where $\Sigma$ is entity of the reference element $T$ such as the edges, faces, or the element itself. So as an example, the set $\mathbb{P}_{2}(\hat{K})$ of polynomials on the reference triangle is:

$$
\mathbb{P}_{2}(\hat{K})=\left\{1, \hat{x}_{1}, \hat{x}_{2}, \hat{x}_{1}^{2}, \hat{x}_{1} \hat{x}_{2}, \hat{x}_{2}^{2}\right\}
$$

Similarly, we denote $\tilde{\mathbb{P}}_{k}(\Sigma)$ as the set of homogeneous polynomials of degree $k$ on $\Sigma$. So for example, the set $\tilde{\mathbb{P}}_{2}(\hat{K})$ of homogeneous polynomials of secondorder on the reference triangle is:

$$
\tilde{\mathbb{P}}_{2}(\hat{K})=\left\{\hat{x}_{1}^{2}, \hat{x}_{1} \hat{x}_{2}, \hat{x}_{2}^{2}\right\}
$$

We now define a new polynomial space $S_{k}(\hat{K})$ as:

$$
S_{k}(\hat{K}):=\left\{\boldsymbol{p} \in[\tilde{\mathbb{P}}(\hat{K})]^{2}: \boldsymbol{p} \cdot \hat{x}=0\right\}
$$

We now have all the necessary components ready to define our finite element $\operatorname{NED}_{1}(\hat{K})$ which will construct a conforming subspace of $H\left(\operatorname{rot}, \Omega_{0}\right)$.

Geometry The geometry is the standard reference triangle $\hat{K} \subset \mathbb{R}^{2}$.

Function Space Nédélec characterised the function space $\mathcal{V}_{\mathrm{NED}_{k}}$ in $\mathbb{R}^{d}$ for $d=$ 2,3 as the polynomial space:

$$
\mathcal{V}_{\mathrm{NED}_{k}}:=\left(\mathbb{P}_{k-1}(\hat{K})\right)^{d} \oplus S^{k}
$$

where the symbol $\oplus$ means the direct sum of the two vector spaces.

In $\mathbb{R}^{2}$ this space can be written in an equivalent form:

$$
\mathcal{V}_{\mathrm{NED}_{k}}=\left(\mathbb{P}_{k-1}(\hat{K})\right)^{2} \oplus \tilde{\mathbb{P}}_{k-1}\left(\begin{array}{c}
-\hat{x}_{2} \\
\hat{x}_{1}
\end{array}\right)
$$


As we will only be deriving the lowest-order element $k=1$ we can write the space $\mathcal{V}_{\mathrm{NED}_{1}}$ as:

$$
\begin{aligned}
\mathcal{V}_{\mathrm{NED}_{1}} & =\left(\mathbb{P}_{0}(\hat{K})\right)^{2} \oplus \tilde{\mathbb{P}}_{0}\left(\begin{array}{c}
-\hat{x}_{2} \\
\hat{x}_{1}
\end{array}\right) \\
& =\left\langle\left(\begin{array}{l}
1 \\
0
\end{array}\right),\left(\begin{array}{l}
0 \\
1
\end{array}\right),\left(\begin{array}{c}
-\hat{x}_{2} \\
\hat{x}_{1}
\end{array}\right)\right\rangle
\end{aligned}
$$

We can therefore write the shape functions $N_{i} \in \mathcal{V}_{\mathrm{NED}_{1}}(\mathbb{R})$ as:

$$
N_{i}=a_{i}+b_{i}\left(\begin{array}{c}
-\hat{x}_{2} \\
\hat{x}_{1}
\end{array}\right)
$$

Degrees of Freedom The set of degrees of freedom $\mathcal{L}$ defined on $\mathcal{V}_{\mathrm{NED}_{1}}$ in the 2 dimensional case consist of two types of linear functionals:

1. edge degrees of freedom on $\hat{e}$

$$
l(\boldsymbol{v})=\int_{\hat{e}}(\boldsymbol{v} \cdot \hat{\boldsymbol{\tau}}) p d \hat{s} \quad \forall p \in \mathbb{P}_{k-1}(\hat{e}) \quad \forall \hat{e} \in \hat{K}
$$

giving a total of $3 k$ edge degrees of freedom.

2. inner degrees of freedom on $\hat{K}$

$$
l(\boldsymbol{v})=\int_{\hat{K}} \boldsymbol{v} \cdot \boldsymbol{p} d \hat{\boldsymbol{x}} \quad \forall p \in\left(\mathbb{P}_{k-2}(\hat{K})\right)^{2} \quad k \geq 2 \text { only }
$$

giving a total of $k(k-1)$ inner degrees of freedom

Fortunately in our case $k=1$ we only have 3 edge degrees of freedom specified by eq. (A.9) and the calculations are relatively simple.

We label the edges as in fig. 3 and orient the unit tangent vectors as shown giving:

$$
\hat{\boldsymbol{\tau}}_{1}=\frac{1}{\sqrt{2}}\left(\begin{array}{c}
-1 \\
1
\end{array}\right), \quad \hat{\tau}_{2}=\left(\begin{array}{l}
0 \\
1
\end{array}\right), \quad \hat{\tau}_{3}=\left(\begin{array}{l}
1 \\
0
\end{array}\right)
$$

We can then write the set of edge degrees of freedom $l_{i}$ using eq. (A.9) as:

$$
l_{i}(\boldsymbol{v})=\int_{\hat{e}_{i}}\left(\boldsymbol{v} \cdot \hat{\boldsymbol{\tau}}_{i}\right) \times 1 d \hat{s} \quad i=1,2,3
$$

The final step is to construct a finite-element basis $N_{1}, N_{2}, N_{3}$. To do this we require:

$$
l_{i}\left(N_{j}\right)=\delta_{i j}
$$

We will perform this calculation for the degree of freedom $l_{1}$ defined across the edge $e_{1}$ as this is the trickiest calculation.

$$
l_{1}\left(N_{j}\right)=\int_{\hat{e}_{1}}\left(a_{j}+b_{j}\left(\begin{array}{c}
-\hat{x}_{2} \\
\hat{x}_{1}
\end{array}\right)\right) \cdot \frac{1}{\sqrt{2}}\left(\begin{array}{c}
-1 \\
1
\end{array}\right) d \hat{s}
$$




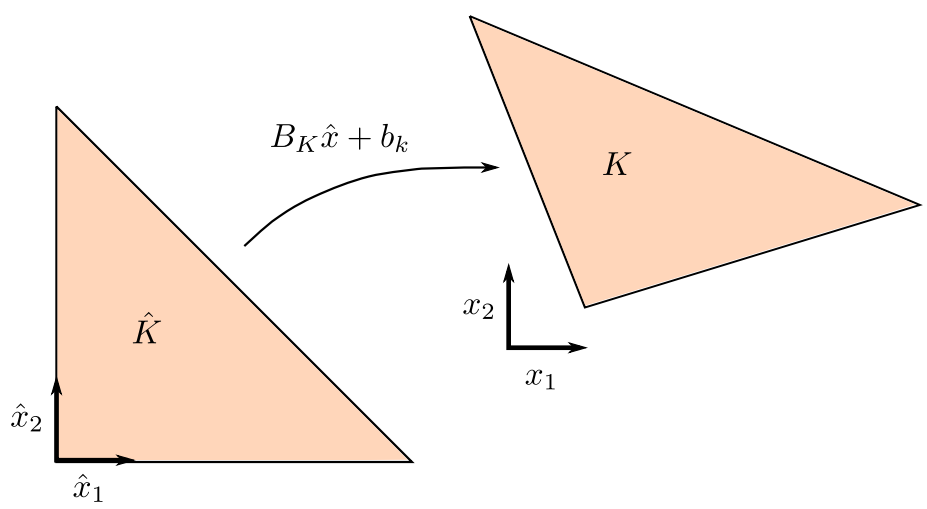

Figure B.16: Transform between reference element $\hat{K}$ and physical element $K$

We then parametrize the edge $\hat{e}_{1}$ as:

$$
\begin{gathered}
r(t)=\langle 1-t, t\rangle, \quad \hat{x}_{1}=1-t, \quad \hat{x}_{2}=t \\
d \hat{s}^{2}=(-d t)^{2}+(d t)^{2}=2 d t^{2}
\end{gathered}
$$

giving the transformed line integral as:

$$
\begin{aligned}
l_{1}\left(N_{j}\right) & =\frac{1}{\sqrt{2}} \int_{t=0}^{t=1}\left(-a_{1 j}+a_{2 j}+b_{j} t+b(1-t)\right) \sqrt{2} d t \\
& =-a_{1 j}+a_{2 j}+b_{j}
\end{aligned}
$$

After repeating the above integration procedure for $l_{2}$ and $l_{3}$ we get the following set of equations for $i=1,2,3$ :

$$
\left[\begin{array}{ccc}
-1 & 1 & 1 \\
0 & 1 & 0 \\
1 & 0 & 0
\end{array}\right]\left(\begin{array}{c}
a_{1 i} \\
a_{2 i} \\
b_{i}
\end{array}\right)=\left[\begin{array}{l|l|l}
1 & 0 & 0 \\
0 & 1 & 0 \\
0 & 0 & 1
\end{array}\right]
$$

Solving gives:

$$
\begin{gathered}
a_{11}=a_{21}=a_{12}=a_{23}=0 \\
b_{1}=a_{22}=a_{13}=b_{3}=1 \\
b_{2}=-1
\end{gathered}
$$




\section{Appendix B. Conforming affine transform}

We define an affine map $F_{k}$ between a general cell $K$ in the global coordinate system $x$ and the reference cell $\hat{K}$ as:

$$
\hat{K} \ni x=F_{K}(\hat{x})=B_{K} \hat{x}+b_{K}
$$

To ensure that we construct an $H(\operatorname{curl} ; \Omega)$ conforming field across the triangulation $\mathcal{T}_{h}$ we must use the covariant vector-field transform. To obtain the element shape functions $N_{i}(x)$ from the reference shape functions $\hat{N}_{i}(\hat{x})$ we use the following covariant transform:

$$
\boldsymbol{N}_{i}(\boldsymbol{x})=\left(\widehat{\boldsymbol{D F}}_{K}^{-T} \hat{\mathbf{N}}_{i}\right) \circ \boldsymbol{F}_{K}^{-1}(\boldsymbol{x})
$$

where $\widehat{D F}_{K} \in \mathbb{R}^{2 \times 2}$ is the Jacobian of the element map:

$$
\widehat{D F}_{K}=\frac{\partial}{\partial \hat{x}} F_{K}(\hat{x})
$$

This transform is the same as that used to transform the gradients of the shape functions in the standard $H^{1}(\Omega)$ conforming finite element methods.

In the case of the affine map defined above the Jacobian is simply a constant for each $K$ :

$$
\widehat{D F_{K}}=B_{K}
$$

[1] D. Chapelle, K. Bathe, The Finite Element Analysis of Shells - Fundamentals, Springer, 2nd edition, 2010.

[2] J. H. Argyris, I. Fried, D. W. Scharpf, The TUBA family of plate elements for the matrix displacement method, The Aeronautical Journal of the Royal Aeronautical Society 72 (1968) 701-709.

[3] O. C. Zienkiewicz, R. L. Taylor, J. M. Too, Reduced integration technique in general analysis of plates and shells, International Journal for Numerical Methods in Engineering 3 (1971) 275-290.

[4] E. N. Dvorkin, K. Bathe, A continuum mechanics based four-node shell element for general non-linear analysis, Engineering Computations 1 (1984) 77-88.

[5] K. Bathe, F. Brezzi, S. W. Cho, The MITC7 and MITC9 plate bending elements, Computers \& Structures 32 (1989) 797-814.

[6] P. Lee, K. Bathe, Development of MITC isotropic triangular shell finite elements, Computers \& Structures 82 (2004) 945-962.

[7] E. L. Wilson, R. L. Taylor, W. P. Doherty, J. Ghaboussi, Incompatible displacement models, Numerical and Computer Methods in Structural Mechanics 43 (1973). 
[8] J. C. Simo, M. S. Rifai, A class of mixed assumed strain methods and the method of incompatible modes, International Journal for Numerical Methods in Engineering 29 (1990) 1595-1638.

[9] K. Bletzinger, M. Bischoff, E. Ramm, A unified approach for shearlocking-free triangular and rectangular shell finite elements, Computers \& Structures 75 (2000) 321-334.

[10] F. Koschnick, M. Bischoff, N. Camprubí, K. Bletzinger, The discrete strain gap method and membrane locking, Computer Methods in Applied Mechanics and Engineering 194 (2005) 2444-2463.

[11] D. N. Arnold, Mixed finite element methods for elliptic problems, Computer Methods in Applied Mechanics and Engineeering (1990) 281-300.

[12] W. Kanok-Nukulchai, W. Barry, K. Saran-Yasoontorn, P. H. Bouillard, On elimination of shear locking in the element-free Galerkin method, International Journal for Numerical Methods in Engineering 52 (2001) 705725 .

[13] O. Garcia, E. A. Fancello, C. S. de Barcellos, C. A. Duarte, $h p$-Clouds in Mindlin's thick plate model, International Journal for Numerical Methods in Engineering 47 (2000) 1381-1400.

[14] P. de T. R. Mendonça, C. S. de Barcellos, A. Duarte, Investigations on the $h p$-Cloud method by solving Timoshenko beam problems, Computational Mechanics 25 (2000) 286-295.

[15] Y. Choi, S. Kim, Bending analysis of Mindlin-Reissner plates by the element free galerkin method with penalty technique, Journal of Mechanical Science and Technology 17 (2003) 64-76.

[16] B. M. Donning, W. K. Liu, Meshless methods for shear-deformable beams and plates, Computer Methods in Applied Mechanics and Engineering 152 (1998) 47-71.

[17] T. Q. Bui, M. N. Nguyen, C. Zhang, A meshfree model without shearlocking for free vibration analysis of first-order shear deformable plates, Engineering Structures In Press, Corrected Proof (2011).

[18] V. Leitão, C. Alves, C. A. Duarte, Advances in Meshfree Techniques, Springer, 1 edition, 2007.

[19] W. Liu, S. Li, T. Belytschko, Moving least-square reproducing kernel methods (I) methodology and convergence, Computer Methods in Applied Mechanics and Engineering 143 (1997) 113-154.

[20] J. M. Melenk, I. Babuska, The partition of unity finite element method: Basic theory and applications, Computer Methods in Applied Mechanics and Engineering 139 (1996) 289-314. 
[21] S. Beissel, T. Belytschko, Nodal integration of the element-free Galerkin method, Computer Methods in Applied Mechanics and Engineering 139 (1996) 49-74.

[22] D. Wang, J. Chen, Locking-free stabilized conforming nodal integration for meshfree Mindlin-Reissner plate formulation, Computer Methods in Applied Mechanics and Engineering 193 (2004) 1065-1083.

[23] J. Cho, S. Atluri, Analysis of shear flexible beams, using the meshless local Petrov-Galerkin method, based on a locking-free formulation, Engineering Computations 18 (2001) 215-240.

[24] Y. Vidal, P. Villon, A. Huerta, Locking in the incompressible limit: pseudo-divergence-free element free Galerkin, Communications in $\mathrm{Nu}$ merical Methods in Engineering 19 (2003) 725-735.

[25] D. González, E. Cueto, M. Doblaré, Volumetric locking in natural neighbour Galerkin methods, International Journal for Numerical Methods in Engineering 61 (2004) 611-632.

[26] J. Simo, R. Taylor, K. Pister, Variational and projection methods for the volume constraint in finite deformation elasto-plasticity, Computer Methods in Applied Mechanics and Engineering 51 (1985) 177-208.

[27] D. P. Recio, R. M. Jorge, L. M. S. Dinis, Locking and hourglass phenomena in an element-free Galerkin context: the B-bar method with stabilization and an enhanced strain method, International Journal for Numerical Methods in Engineering 68 (2006) 1329-1357.

[28] A. Ortiz, M. Puso, N. Sukumar, Maximum-entropy meshfree method for compressible and near-incompressible elasticity, Computer Methods in Applied Mechanics and Engineering 199 (2010) 1859-1871.

[29] A. Ortiz, M. Puso, N. Sukumar, Maximum-entropy meshfree method for incompressible media problems, Finite Elements in Analysis and Design 47 (2011) 572-585.

[30] M. Arroyo, M. Ortiz, Local maximum-entropy approximation schemes: a seamless bridge between finite elements and meshfree methods, International Journal of Numerical Methods in Engineering 65 (2006) 2167-2202.

[31] N. Sukumar, R. W. Wright, Overview and construction of meshfree basis functions: from moving least squares to entropy approximants, International Journal of Numerical Methods in Engineering 70 (2007) 181-205.

[32] P. A. Raviart, J. M. Thomas, A mixed finite element method for 2-nd order elliptic problems, in: I. Galligani, E. Magenes (Eds.), Mathematical Aspects of Finite Element Methods, volume 606, Springer Berlin Heidelberg, 1977, pp. 292-315. 
[33] J. C. Nedelec, Mixed finite elements in $\mathbb{R}^{3}$, Numerische Mathematik 35 (1980) 315-341.

[34] C. E. Shannon, A mathematical theory of communication, The Bell Systems Technical Journal 27 (1948) 379-423.

[35] E. T. Jaynes, Information theory and statistical mechanics. II, Physical Review 108 (1957) 171-190.

[36] S. A. Frank, Measurement scale in maximum entropy models of species abundance, Journal of Evolutionary Biology 24 (2011) 485-496.

[37] A. Ratnaparkhi, Learning to parse natural language with maximum entropy models, Machine Learning 34 (1999) 151-175.

[38] N. Sukumar, Construction of polygonal interpolants: a maximum entropy approach, International Journal for Numerical Methods in Engineering 61 (2004) 2159-2181.

[39] N. Sukumar, Maximum entropy approximation, AIP Conference Proceedings 803 (2005) 337-344.

[40] T. Belytschko, Y. Y. Lu, L. Gu, et al., Element-free galerkin methods, International Journal for Numerical Methods in Engineering 37 (1994) 229-256.

[41] N. Sukumar, R. J. Wets, Deriving the continuity of Maximum-Entropy basis functions via variational analysis, SIAM Journal on Optimization 18 (2007) 914-925.

[42] S. Fernández-Méndez, A. Huerta, Imposing essential boundary conditions in mesh-free methods, Computer Methods in Applied Mechanics and Engineering 193 (2004) 1257-1275.

[43] J. Nitsche, Über ein variationsprinzip zur lösung von DirichletProblemen bei verwendung von teilräumen, die keinen randbedingungen unterworfen sind, in: Abhandlungen aus dem Mathematischen Seminar der Universität Hamburg, volume 36, p. 9-15.

[44] T. Belytschko, D. Organ, Y. Krongauz, A coupled finite element-elementfree Galerkin method, Computational Mechanics 17 (1995) 186-195.

[45] C. J. Cyron, M. Arroyo, M. Ortiz, Smooth, second order, non-negative meshfree approximants selected by maximum entropy, International Journal of Numerical Methods in Engineering 79 (2009) 1605-1632.

[46] D. González, E. Cueto, M. Doblaré, A higher order method based on local maximum entropy approximation, International Journal for Numerical Methods in Engineering 83 (2010) 741-764. 
[47] A. Rosolen, D. Millán, M. Arroyo, On the optimum support size in meshfree methods: A variational adaptivity approach with maximum-entropy approximants, International Journal for Numerical Methods in Engineering 82 (2010) 868-895.

[48] D. Millán, A. Rosolen, M. Arroyo, Thin shell analysis from scattered points with maximum-entropy approximants, International Journal for Numerical Methods in Engineering 85 (2011) 723-751.

[49] L. L. Yaw, N. Sukumar, S. K. Kunnath, Meshfree co-rotational formulation for two-dimensional continua, International Journal for Numerical Methods in Engineering 79 (2009) 979-1003.

[50] S. Boyd, L. Vandenberghe, Convex Optimization, Cambridge University Press, 2004.

[51] D. Braess, Finite Elements: Theory, Fast Solvers, and Applications in Solid Mechanics, Cambridge University Press, 3 edition, 2007.

[52] S. M. Alessandrini, D. N. Arnold, R. S. Falk, R. L. Madureira, Derivation and justification of plate models by variational methods, in: M. Fortin (Ed.), Plates and Shells, American Mathematical Society, 1999, pp. 1-20.

[53] D. N. Arnold, R. S. Falk, Asymptotic analysis of the boundary layer for the Reissner-Mindlin plate model, SIAM Journal on Mathematical Analysis 27 (1996) 486-514.

[54] P. D. Ledger, K. Morgan, O. Hassan, Electromagnetic scattering simulation using an $H$ (curl) conforming $h p$ finite element method in three dimensions, International Journal for Numerical Methods in Fluids 53 (2007) 1267-1296.

[55] A. Iosilevich, K. Bathe, F. Brezzi, On evaluating the inf-sup condition for plate bending elements, International Journal for Numerical Methods in Engineering 40 (1997) 3639-3663.

[56] N. Sukumar, Fortran 90 library for maximum-entropy basis functions, 2008-.

[57] J. G. Wang, G. R. Liu, A point interpolation meshless method based on radial basis functions, Int. J. Numer. Meth. Eng 54 (2002) 1623-1648.

[58] D. Abrahams, Boost Python C++ library, 2011-.

[59] T. E. Oliphant, Guide to NumPy, 2006.

[60] A. Logg, G. N. Wells, DOLFIN: automated finite element computing, ACM Transactions on Mathematical Software (TOMS) 37 (2010) 20. 
[61] R. C. Kirby, Algorithm 839: FIAT, a new paradigm for computing finite element basis functions, ACM Transactions on Mathematical Software 30 (2004) 502-516.

[62] E. Jones, T. Oliphant, P. Peterson, et al., SciPy: Open source scientific tools for Python, 2001-.

[63] J. Reddy, Theory and Analysis of Elastic Plates and Shells, CRC Press, 2 edition, 2006.

[64] C. E. İmrak, İ. Gerdemeli, An exact solution for the deflection of a clamped rectangular plate under uniform load, Applied Mathematical Sciences 1 (2007) 2129-2137.

[65] A. Buffa, G. Sangalli, R. Vázquez, Isogeometric analysis in electromagnetics: B-splines approximation, Computer Methods in Applied Mechanics and Engineering 199 (2010) 1143-1152.

[66] P. G. Ciarlet, The Finite Element Method for Elliptic Problems, SIAM: Society for Industrial and Applied Mathematics, 2nd edition, 2002. 